\title{
Sur les droits du travail en Amérique latine. Évolution et perspectives
}

Adrián Goldin

\section{(2) OpenEdition}

1 Journals

Édition électronique

URL : https://journals.openedition.org/annuaire-cdf/16372

DOI : $10.4000 /$ annuaire-cdf. 16372

ISBN : 978-2-7226-0572-5

ISSN : 2109-9227

Éditeur

Collège de France

Édition imprimée

Date de publication : 30 décembre 2020

Pagination : 696-697

ISBN : 978-2-7226-0516-9

ISSN : 0069-5580

\section{Référence électronique}

Adrián Goldin, «Sur les droits du travail en Amérique latine. Évolution et perspectives », L'annuaire du

Collège de France [En ligne], 118 | 2020, mis en ligne le 01 avril 2021, consulté le 22 août 2022. URL

http://journals.openedition.org/annuaire-cdf/16372 ; DOI : https://doi.org/10.4000/annuaire-cdf. 16372 


\title{
SUR LES DROITS DU TRAVAIL EN AMÉRIQUE LATINE. ÉVOLUTION ET PERSPECTIVES
}

\author{
Adrián GOLDIN \\ Professeur émérite à l'université de Buenos Aires et de San Andrés (Argentine) \\ et professeur honoraire de la Société internationale de droit du travail \\ et de la sécurité sociale
}

Mots-clés : droit du travail, Amérique latine

Conférence prononcée le 14 mai 2018 (invitation sur proposition du Pr Alain Supiot), disponible en audio sur le site internet du Collège de France : https://www.collegede-france.fr/site/alain-supiot/guestlecturer-2017-2018_1.htm.

J'ai tenté d'identifier certaines caractéristiques communes des droits du travail en Amérique latine. Cela ne signifie pas selon moi qu'il existe une entité qui peut être caractérisée sans réserve comme un droit du travail latino-américain, question qui mérite une discussion plus approfondie; il s'agit simplement d'un effort méthodologique pour présenter de manière synthétique un très large ensemble de régimes juridiques qui seraient autrement très difficiles à considérer avec un certain ordre dans cette brève présentation.

En tout cas, il faudrait dire que les droits du travail de l'Amérique latine partagent une appartenance commune au système juridique continental. Selon cette condition, la réglementation du travail de l'Amérique latine exprime un droit fondé sur la loi comme première expérience juridique, l'idée d'ordre public social, une intervention étatique active, la prédominance de la norme impérative. Par conséquent, celle-ci ne peut pas être déplacée au détriment du travailleur, dans le cadre d'un système de sources qui est un mélange, en différents dosages dans chacune de ces nations, des lois et des conventions collectives qui, dans leur ensemble, constituent un système complexe de techniques pour limiter l'autonomie de la volonté.

Cependant, je suis d'avis que ces droits expriment une appartenance faible au modèle social européen. C'est une circonstance qui, pourrait-on dire, les a rendus plus sensibles aux compulsions des marchés qui ont eu leur implantation la plus forte dans les années 1990, donc à son impact disproportionné, l'introduction conséquente de réformes biaisées et fragiles et, même, la propension aux restaurations anachroniques subséquentes qui ont eu lieu dans certains de ces pays à partir des premières années du nouveau millénaire. Il faut dire, cependant, que peutêtre précisément à cause de sa propre fragilité, ces processus n'ont pas réussi à déplacer les fondements structurels des droits sociaux de l'Amérique latine, pas même pendant la période où les transformations les plus profondes se sont matérialisées.

Dans ce cadre, la région présente - au moins dans la littéralité des textes de la législation - un niveau de protection élevé par rapport à d'autres régions, et même à 
celles qui ont influencé leur configuration, mais, en même temps, une couverture plus faible qui a, parmi ses facteurs déterminants et ses expressions les plus significatives, la grande dimension du secteur informel, une culture étendue de l'anomie, l'absence de ressources de contrôle (ou le manque d'intérêt à les établir), l'attribution de plus d'importance à l'existence de la loi qu'à son application effective.

Une des différences significatives qui distinguent les droits du travail des nations latino-américaines par rapport à d'autres appartenant à des pays en voie de développement est que les premiers sont des droits influencés qui, comme nous venons de le mentionner, expriment la tradition juridique héritée de l'Europe continentale, mais ils ne sont pas des droits transplantés. La plupart de ces pays ont accédé à leur indépendance dans les premières décennies du XIX ${ }^{\mathrm{e}}$ siècle et, par conséquent, avant le développement des processus d'industrialisation qui ont déterminé la naissance des droits du travail modernes.

Dans ce contexte, l'influence des Conventions et Recommandations de l'OIT sur les systèmes latino-américains a été - et demeure - considérable. À son tour, la relation fréquente entre la législation tutélaire et les régimes autoritaires qui ont prévalu de façon récurrente dans la région ne semble pas avoir modifié ces profils tutélaires, du moins en ce qui concerne les normes qui régissent les relations individuelles : parfois populistes, parfois paternalistes, en Amérique latine, l'État autoritaire était aussi un État protecteur.

D'autre part, il semble pertinent de souligner deux processus additionnels qui ont beaucoup contribué à la configuration des droits actuels du travail latino-américains : le processus de codification et celui de constitutionnalisation et d'exaltation conséquente des droits fondamentaux de la personne. Il faut ajouter que dans les dernières décennies, tandis que les lois ont eu dans la région une tendance à remettre en cause le Droit du travail, les constitutions approuvées à la même époque avaient une tendance à le renforcer.

À son tour, et par rapport aux schémas de régulation des relations collectives de travail qui l'emportent dans la région, on pourrait dire qu'à l'exception notable de l'Uruguay, ils partagent tous un biais contrôlant et restrictif, dans de nombreux cas de lignage autoritaire, mais répondant au moins à deux modèles très divers : la majorité, celui du contrôle par le biais de la décentralisation imposée par la loi ; pour le reste - ce sont notamment les cas de l'Argentine et du Brésil, qui ont reçu des influences provenant des régimes autoritaires européens de la première moitié du siècle dernier -, celui du contrôle par centralisation aussi imposée par décision de la loi. Les deux modèles sont loin d'être alignés avec les principes de la liberté syndicale. Il faut dire cependant que, dans de nombreux cas, ils ont subi au cours des dernières décennies des changements précieux - induits par la logique démocratique survenant - qui les auraient poussés (sans l'atteindre complètement) vers une plus étroite proximité avec les Conventions 87 et 98 de l'OIT.

Enfin, les développements plus récents des droits du travail latino-américains sont décrits, en considérant, entre autres, les tendances de destandardisation des formes de l'utilisation du travail, de l'affaiblissement subjectif du système de protection du travail et de l'extension de la protection sociale par moyen de l'universalisation de sa couverture. En même temps, et corrélativement, on a noté les tendances vers la segmentation et la pluralisation des régimes de protection du travail et d'autres développements récents. 